\title{
A Nonparametric Scale Analysis of the Development of Conservation
}

\author{
Johannes Kingma and Elisabeth $M$. TenVergert \\ University of Alberta
}

\begin{abstract}
The purpose of this study was to investigate the development of conservation by using the nonparametric Mokken scale analysis. Subjects were 801 children from kindergarten and primary school Grades 1 and 2 who completed 13 conservation tasks derived from Piaget's publications. It was shown that some selections (i.e., seven, eight, and nine tasks, respectively, at three successive administrations with three-month's interval) formed strong Mokken scales, which were invariant for different samples at the same point in time of test administration. Furthermore, it was found that during the course of development the number of tasks which fitted on the scale increased. However, some reversals of the relative positions of a small number of tasks were found for the scales at different points in time of test administration. It was concluded that application of nonparametric Mokken scale analysis resulted in a new, but very useful instrument for analyzing the order of acquisition of conservation.
\end{abstract}

Since the early $1960 \mathrm{~s}$ a continuous increase in the number of conservation studies can be observed in the developmental psychology literature (Brainerd, 1978). Concerning the standard Piagetian conservation tasks for assessing the presence of conservation, a child is shown two objects that are perceptually identical and equivalent with respect to some quantitative property (e.g., length, weight, volume, etc.). One of the objects is altered per-

APPLIED PSYCHOLOGICAL MEASUREMENT

Vol. 9, No. 4, December 1985, pp. 375-387

(C) Copyright 1985 Applied Psychological Measurement Inc.

0146-6216/85/040375-13\$1.90 ceptually but not quantitatively. Subsequently, the experimenter then determines whether or not the child understands that quantitative equivalence is conserved across the perceptual transformation.

According to Piaget and his associates, a child's ability to conserve shows a gradual development through different concept areas (number, length, weight, etc.) in which some sequence can be distinguished, for example, the acquisition of number conservation precedes conservation of quantity (Piaget, 1952; Piaget \& Szeminska, 1941), whereas conservation of substance precedes weight conservation, which, in turn precedes volume conservation (Piaget \& Inhelder, 1941). Furthermore, Piaget, Inhelder, and Szeminska (1948) reported that length conservation preceded conservation of perimeter, and the latter preceded conservation of surface, whereas conservation of volume was acquired after conservation of surface.

These statements about the sequence of development of conservation might lead to the conclusion that these conservation tasks (from different concept areas) might provide a kind of developmental scale for assessing a child's level of conservation. However, notwithstanding the enormous quantity of conservation research, such a developmental scale has not been developed. The purpose of the present study was to investigate whether a broad range of conservation tasks in different concept areas could provide a developmental scale for the acquisition of conservation. 
In test construction, techniques such as item analysis and factor analysis are generally used (see Nunnally, 1978). When it appears that a test is sufficiently homogeneous (i.e., all items measure the same thing), then the total score of a child can be compared with the mean total score of children at the same age level. In this way an individual child's level of performance on certain ability tasks can be compared to the mean performance of other children in the same or different age groups. Such comparisons become especially important when constructing a developmental psychological scale that can be used to obtain an indication of whether a child shows a retardation or whether he/she is precocious in the acquisition of an ability, and so forth. Therefore, it is necessary that the items (which measure the same underlying construct) will be ordered according to, for example, increasing difficulty level.

One of the scaling methods relevant for developmental psychology is Guttman scale analysis (Goodman, 1959; Guttman, 1950). This method has been used by Kingma (1984a), Siegel (1971), Michie (1985), and Wohlwill (1960) to investigate the development of number concept, whereas Kofsky (1966) used this method to study the order of acquisition of classificatory skills. However, this method has some disadvantages, because the model is deterministic, and this assumption has been shown to be rather unrealistic (Mokken, 1971; Nunnally, 1978).

Guttman scale analysis can be seen as a precursor of modern item response theory (IRT) of which Mokken scale analysis is an example (see Hambleton \& van der Linden, 1982). With Mokken scale analysis (Mokken, 1971; Mokken \& Lewis, 1982) an ordering of both items and persons can be made, just as with Guttman scale analysis.

In contrast to the Guttman model, the Mokken model is stochastic, that is, item responses are considered to be outcomes of stochastic processes which can be characterized by certain parameters. By using the Mokken model, these probabilities can be written as a function of a person parameter and an item parameter, $\pi(\theta, \delta)$. Therefore, the advantage of using this model is that the restrictions placed on the data are less stringent than those posed by the Guttman model.

\section{The Mokken Model}

The Mokken model is a nonparametric elaboration of a type of model implied by most of the current parametric latent trait models (see Mokken \& Lewis, 1982). It is assumed that a one-dimensional latent attribute (e.g., conservation, classification, etc.) exists on an underlying continuum. Furthermore, it is assumed that each person has a certain unknown value on this continuum, which is represented by a person's parameter $\theta$. The items related to the attribute need to be homogeneous with respect to it. For the analysis, dichotomous items are used in which one alternative is designated as "positive" with respect to the latent ability of interest.

In a developmental scale, the person's parameter $\theta$ represents how far the individual has progressed in the acquisition of the ability as measured by the scale. In addition, an item parameter $\delta$ is introduced. The $\delta$ of an item represents the point of development in the acquisition of the ability as measured by that item. With the use of the Mokken scale, both items and persons can be ordered, that is, the items according to increasing $p$ values (proportion correct response) and persons according to increasing scale score (i.e., the total score on the set of items). The scale score serves as an estimator of the person's $\theta$ (eventually divided by the total number of items so that a value between 0 and 1 will always-be obtained). The $p$ value of an item provides an estimate of the $\delta$ of an item.

Since the Mokken model assumes unidimensional measurement, the characteristics of this stochastic cumulative model are: (1) a one-dimensional latent variable on which each person has an unknown value $\theta$; (2) the probability of a correct response increases monotonically over $\theta$; (3) the items have different difficulties; (4) their value $\delta$ on the latent variable equals the probability $\pi(\theta, \delta)$ $=.50$ of a person's correct response and (5) the item characteristic curves (ICCs) do not intersect. 


\section{Assumptions}

In methods of scaling, an assumption is made about ICCs or item trace lines (see Nunnally, 1978). An ICC is a curve which represents the probability that a person gives a correct response to an item, as a function of the individual's knowledge (competence) of the attribute measured by that item. In the case of conservation, an ICC represents the probability that a child gives a correct response to an item as a function of the child's acquisition of the conservation ability.

According to the Mokken model (Mokken, 1971; Mokken \& Lewis, 1982), the ICCs must meet two assumptions of double monotony (also called the assumption of local independence, see Mokken \& Lewis, 1982). First, the ICCs have to be monotonically increasing in $\theta$, that is, for all persons $i$ and $j$ with $\theta_{i}<\theta_{j}$, the assumption that the probability of giving a correct response on that item by person $j$ will be greater than the probability of giving a correct response by person $i$ on that item must be valid.

A second assumption is that the ICCs have to be monotonically decreasing in $\delta$, that is, for all pairs of items $i$ and $j$ where $i<j$ and $\delta_{i}>\delta_{j}$, the assumption means that for all persons, the probability of a correct response on item $i$ is smaller than the probability of a correct response on item $j$ must be valid (i.e., this assumption implies that an intersection of the ICCs is not allowed). The $\delta$ of an item has been defined as the $\theta$ of a person who has a probability of .50 of giving a correct response to that item. When the two items $i$ and $j$ meet these two assumptions of double monotony, then it follows that $p_{i}<p_{j} \leftrightarrow \delta_{i}>\delta_{j}$.

\section{Checks for Double Monotony}

The Mokken model provides the opportunity to determine whether or not the data contradict the model through inspection of the $\mathbf{P}$ and $\mathbf{P}_{0}$ matrices. If the items are ordered according to increasing $p$ values in a matrix and the proportion of correct responses of each pair of items $i$ and $j$, their $\pi_{i j}$ $(1,1)$, are inserted into this matrix, then these $\pi_{i j}$
$(1,1)$ s should increase from left to right and from top to bottom. Such a matrix is called the $\mathbf{P}$ matrix.

The $\mathbf{P}_{0}$ matrix can be obtained as follows: the items are ordered according to decreasing difficulty in a matrix, and for each item pair $i$ and $j$ the proportion of incorrect responses on both items, their $\pi_{i j}(0,0)$, are inserted. In this matrix, the proportion $\pi_{i j}(0,0)$ should decrease from left to right and hence, also from top to bottom (see Mokken, 1971, pp. 132-183). For the purpose of constructing a scale, as in the present study, only those items are deleted which violate these two assumptions at double monotony. The remaining items order all persons similarly when it is shown that they meet a number of scalability criteria to test the goodness of fit of the Mokken model for the selected items.

\section{Coefficients of Scalability}

Three different coefficients of scalability have been defined: $H_{i j}$ for each pair of items in the scale, $H_{i}$ for each item in the scale with respect to all other items in the scale, and $H$ for the scale as a whole. The coefficient $H_{i j}$ for each item pair has been defined as follows:

$H_{i j}=\frac{p_{i j}-p_{i} p_{j}}{\left(1-p_{j}\right) p_{i}}$,

where $p_{i}<p_{j}$.

This coefficient of reproductivity, also called the homogeneity coefficient (Loevinger, 1948), represents the probability, given a correct response on a difficult item, that a correct response will also be obtained on the easier item (corrected for differences between the probabilities of a correct response of the two items). Furthermore, $p_{i j}$ represents the proportion of persons who give a correct response on both items $i$ and $j$, and $p_{i}$ and $p_{j}$ are the proportion of correct responses on item $i$ and $j$, respectively. Thus, $H_{i j}$ indicates the association between items $i$ and $j$.

A second criterion for scalability is the coefficient $H_{i}$, which is a measure of homogeneity for each item $i$ with respect to the whole set of items. Mokken (1971) defined the coefficient $H_{i}$ (also called the time coefficient of item $i$ ) as: 


$$
H_{i}=\frac{\sum_{i=1}^{k} \sum_{j=i+1}^{k} p_{i}\left(1-p_{j}\right) \cdot H_{i j}}{\sum_{j=1}^{k}\left(1-p_{i}\right) p_{j}+\sum_{j=i+1}^{k}\left(1-p_{i}\right) p_{j}},
$$

where $p_{1}<p_{2}<, \ldots,<p_{k-1}<p_{k}$. This coefficient provides information about whether or not a certain item fits into the scale. Finally, a coefficient of scalability (i.e., a coefficient of homogeneity) can similarly be defined for a set of $k$ items as a whole. This coefficient was defined by Loevinger (1947) and Mokken (1971) as a normalized weighted sum over the $H_{i j}$ for all item pairs:

$$
H=\frac{\sum_{i=1}^{k} \sum_{j=i+1}^{-1} p_{i}\left(1-p_{j}\right) \cdot H_{i j}}{\sum_{i=1}^{k} \sum_{j=i+1}^{k} p_{i}\left(1-p_{j}\right)},
$$

where $p_{1}<p_{2}<, \ldots,<p_{k}$. When items do not measure anything in common, then as a consequence $H_{i j}, H_{i}$, and $H$ are all zero. The maximum of $H_{i j}$ is 1.0 .

\section{Criteria for Scalability}

In summary, a set of items together form a Mokken scale if the following conditions are satisfied. First, the ICCs must meet the assumptions of double monotony. As a check, the $\mathbf{P}$ matrix and the $\mathbf{P}_{0}$ matrix should be inspected for monotony (deviations of about .03 are allowed). Second, $H_{i j} \mathrm{~s}$ must be greater than zero. Third, all $H_{i} \mathrm{~s}$ must be greater than or equal to a positive constant (chosen by the researcher), $H_{i}>c$. In addition, $H$ and all $H_{i} \mathrm{~s}$ should be significantly greater than 0 according to a level of confidence chosen by the researcher (see Mokken, 1971).

Mokken (1971, p. 185) proposed the following classification of scales in terms of $H$ : (1) $.50<H$, a strong scale; (2) $.40<H<.50$, a medium scale; (3) $.30<H<.40$, a weak scale; and (4) $.30>H$, no scale. Thus, by using the coefficients $H_{i j}, H_{i}$, and $H$, and the $\mathbf{P}$ matrix and the $\mathbf{P}_{0}$ matrix as a check for double monotony, the goodness of fit of the Mokken model can be tested.

In practice, however, when constructing a new scale, some of the items in the set may not meet all these criteria. Those items that do not satisfy one of these criteria are omitted. For the remaining ones, the procedure of checking the criteria is repeated until a real stochastic Mokken scale is found. The computer program Mokken Scale (Niemoller, Verschuur, \& Stokman, 1980) ${ }^{1}$ provides such a search procedure.

\section{Robustness}

Once a scale has been found, the Mokken model can also be used for testing the scale on different samples. When using different samples, the robustness (or invariance) of the scale can be determined for comparing the values of $H$ and $H_{i}$ for these samples. Mokken has developed the test statistic $T$, which is used to test the null hypothesis that the values of $H_{h}$ for subgroups $H_{h}(h=1,2$, $\ldots, p)$ are equal, that is, $H_{1}=H_{2}=H_{3}=, \ldots$, $=H_{h}=, \ldots, H_{p}=H$. This statistic $T$ is defined as:

$T=\sum_{h=1}^{p} \frac{\left(\hat{H}_{h}-\bar{H}\right)^{2}}{S^{2}\left(\hat{H}_{h}\right)}$,

Under the null hypothesis, $T$ very closely approaches the chi-square distribution with $p-1$ degrees of freedom when $n$ is large. If the null hypothesis cannot be rejected ( $T$ smaller than a critical value, dependent on both the number of degrees of freedom and on a significance level alpha), the mean value $\bar{H}$ is considered a pooled estimate of the estimated values $\hat{H}_{h}$ for the $p$ populations (for definitions of both $\hat{H}_{h}$ and $\bar{H}$, see Mokken, 1971).

In practice, however, as in the majority of models of scale construction, the estimation of $\delta$ of an item will be far more accurate than estimating the $\theta$ of an individual, because the number of subjects in general will be larger than the ordering of items. Thus, due to a relatively small number of items, the ordering of the persons will be less accurate than the ordering of the items. Therefore, the focus of interest in the present study was the ordering of

'Both the Mokken scale analysis and the Mokken test programs are available for Apple II, II plus and IIe under CPM 2.2. operating system. A listing of these programs in Pascal MT + and a floppy disk may be requested from the first author. Versions for CPM68K and IBM microcomputers are in preparation. 
the conservation items in order to obtain a developmental scale for conservation.

\section{Why Use the Mokken Model?}

The question remains as to why use a nonparametric Mokken model when parametric IRT models are already available in the psychological literature. Of course, parametric models possessing the properties (both with item selection-free parameter estimates) have a number of advantages compared to the nonparametric Mokken model. As a consequence, it may be worthwhile to construct developmental psychological instruments of measurement based on such models and to show reasonable empirical fit to these models (see Mokken, 1971). However, it can be argued that in vast areas of social research the application of parametric models may be often too far-fetched. It is well-known that their application presupposes a relatively deep insight into the structure of the variable to be measured and the properties of the items by which it can be measured. This can usually be achieved only in the more advanced stages of research by means of a series of continuous studies of scale evaluation and construction.

Notwithstanding the enormous amount of research about Piagetian concepts in developmental psychology since the early 1960s, the technology of measurement is rather undeveloped in this area (e.g., the lack of developmental scales for these concepts). Therefore, in this field the investigator is engaged in more intermediate or exploratory phases of research (see for a discussion, Brainerd, 1985;
Kingma \& Roelinga, 1984; Rushton, Brainerd, \& Pressley, 1984). For these reasons, it seems legitimate to try to find starting points for scaling models that do not rely too heavily upon specific parametric assumptions (e.g., since these lead to procedures of inference and estimation that are too pretentious and intricate for the level of information, and the precision that can be claimed for the data used in actual measurement). In the present study Mokken scale analysis was used to investigate whether a scale could be constructed from a set of 13 conservation tasks (derived from Piaget's publications).

\section{Method}

\section{Subjects}

Subjects were 801 Dutch children from eight kindergarten and six primary schools in Grades 1 and 2 . These 801 children were divided into four groups. Group 1 (called the longitudinal group) consisted of 178 children who completed the tasks on three occasions during one school year, respectively, in September, January, and April. The remaining 623 children were divided into three groups. Group 2 consisted of 206 children who were administered the tasks in September (called the September group); Group 3 consisting of 204 children who completed the tasks in January (called the January group); and Group 4 consisted of 213 children who completed the tasks in April (called the April group). Table 1 shows the mean ages of these four groups, which were divided into five age lev-

Table 1

Number of Subjects and Their Mean Ages in Months for Each of the Four Samples $(\mathrm{N}=801)$

\begin{tabular}{|c|c|c|c|c|c|c|c|c|c|c|c|}
\hline \multirow[b]{3}{*}{ Sample } & \multirow[b]{3}{*}{ Total N } & \multicolumn{10}{|c|}{ Age Level } \\
\hline & & \multicolumn{2}{|c|}{4} & \multicolumn{2}{|r|}{5} & \multicolumn{2}{|c|}{ older 5} & \multicolumn{2}{|r|}{6} & \multicolumn{2}{|c|}{7} \\
\hline & & $\overline{\mathrm{N}}$ & Mean & $\bar{N}$ & Mean & $\overline{\mathrm{N}}$ & Mean & $\overrightarrow{\mathrm{N}}$ & Mean & $\bar{N}$ & Mean \\
\hline Longitudinal & 178 & 22 & 54.1 & 31 & 62.7 & 32 & 68.5 & 43 & 78.0 & 50 & 90.6 \\
\hline September & 206 & 31 & 54.2 & 33 & 63.5 & 39 & 68.9 & 46 & 78.1 & 57 & 91.6 \\
\hline January & 204 & 32 & 56.1 & 35 & 64.0 & 36 & 69.2 & 45 & 77.4 & 56 & 90.7 \\
\hline April & 213 & 43 & 55.4 & 34 & 62.9 & 40 & 69.0 & 46 & 78.4 & 50 & 91.5 \\
\hline
\end{tabular}

Note: The mean ages of respectively, the September, January and April groups were computed at the time of the first test administration of the longitudinal study. 
els: 4-year-olds, 5-year-olds, older 5-year-olds, 6-year-olds, and 7-year-olds.

\section{Materials}

Before the 13 conservation tasks were administered, an introductory trial was given to accustom the child to the questions. In this trial two identical parallel rows each with six blocks were presented. The experimenter asked the child whether these two rows had the same number of blocks. Following this, two blocks were added to one row and the experimenter asked again whether or not these two rows had the same number of blocks.

Subsequently, the 13 conservation tasks were administered in a random order. The procedure for each task was similar. At first, two identical objects were presented and the child was asked whether these objects were the same for the relevant feature (e.g., length, surface, etc.). Following this, a transformation of one of these two objects was performed, and finally, the child was asked whether or not the transformed object was the same as the nontransformed one for that feature. The following tasks were completed.

A. Conservation of surface. Two identical square folders were presented. One folder, which had been placed as a triangle, was cut across the diagonal into two pieces. The child was asked whether or not the square folder or the triangle had an equal amount of grass.

B. Conservation of number. In this task two identical parallel rows of plastic circles were presented. One row contained six red circles and the other row contained six white circles. Subsequently, the elements of the row with the red circles were placed further apart (see Piaget \& Szeminska, 1941, chp. 2).

C. Conservation of distance. Two pairs of identical towers (blocks) were presented. The lengths (distances) between each pair of towers were identical. A screen was placed between one pair of the towers (see Piaget et al., 1948, chp. 3).
D. Conservation of substance. Two identical clay balls were shown. One ball was transformed into a sausage of about $20 \mathrm{~cm}$ long (see Piaget \& Inhelder, 1941, chp. 1).

E. Conservation of perimeter. On each of the two identical cardboard squares three pins were attached. Around these pins a rope was fastened. Following this, one pin on the top of one triangle was shifted. The experimenter asked whether or not the same distance had to be walked along the two different shaped triangles (see Vingh Bang, 1965).

F. Conservation of quantity. Two identical glasses, each with $150 \mathrm{ml}$ of water, were presented. The contents of one glass were poured into a large glass with diameter $7 \mathrm{~cm}$ and height $9.5 \mathrm{~cm}$ (see Piaget \& Szeminska, 1941).

G. Conservation of discontinuous quantity. The contents of one of the two identical glasses, each with $200 \mathrm{ml}$ of grains, were poured into a long and narrow glass with diameter $3.7 \mathrm{~cm}$ and height $13.5 \mathrm{~cm}$ (see Piaget \& Szeminska, chp. 2).

H. Conservation of weight. Two identical clay balls were shown. One of the two balls was transformed into a pancake with a diameter of about $10 \mathrm{~cm}$ (see Piaget \& Inhelder, 1941, chp. 2).

I. Conservation of covered surface. Two identical green cardboard squares $(20 \times 20 \mathrm{~cm})$, representing two meadows, were shown. Following this, two identical yellow folders (each $10 \times 10 \mathrm{~cm}$ ), representing potato fields, were shown. The folders were placed into the meadows. Subsequently, one of the yellow folders was cut across the diagonal and the two pieces were put on the green cardboard in the form of a triangle. The child was asked whether or not the two meadows had the same amount of grass (see Piaget et al., 1948).

J. Conservation of length. Two identical cardboard strips were presented. One strip was transformed into a W shape (see Piaget et al., 1948).

K. Conservation of discontinuous length. Two rows of five matches each were laid down. 
In one row two match sticks were broken in two and one match stick at the end of that row was turned 90 degrees (see Piaget et al., 1948, chps. 4 \& 5).

L. Conservation of geometric volume. One blue cardboard square $(40 \times 40 \mathrm{~cm})$, representing a lake, was shown. Four identical green folders were laid down close together on the blue cardboard, forming an island. On this island two identical towers were built (each consisting of three identical blocks of $6 \times 6 \times$ $6 \mathrm{~cm}$ ). Following this, the experimenter told the child that a heavy storm damaged the island and one of the towers, and that the people who lived in this tower had then built three houses (each consisting of one block of the tower). Finally, the child was asked whether or not the three houses together had the same space as the tower (see Piaget et al., 1948, chps. $13 \& 14$ ).

M. Conservation of physical volume. Two identical containers, each filled with an equal amount of water, were presented. The level of the water was marked by a rubber band. Following this, two identical clay balls were shown. One ball was put into one container and the rise of the water level was marked with a sticker. Subsequently, the other ball was transformed into a pancake. Finally, the child was asked whether or not the water in the other container would rise to the same level as it did with the clay ball when the pancake was put into the container (see Piaget \& Inhelder, 1941, chp. 12).

In order to avoid a response set, due to the fact that two identical objects were used in all 13 conservation tasks, two conservation tasks were also administered (between tasks $\mathrm{E}$ and $\mathrm{F}$, and between tasks $I$ and $J$ ) in which the two objects were unequal.

\section{Scoring}

For the 13 conservation tasks, the scoring system of the well-known Conservation Assessment Kit (Goldschmid \& Bentler, 1968) was used. Accord- ing to this, a conservation task was given a score of 1 when the child gave both a correct judgment and a correct justification for that judgment belonging to the following categories: (1) identity (you did not add or subtract anything, or it is still the same clay, etc.), (2) reversibility (you can change again the sausage into the ball, etc.), and (3) compensation (the sausage is longer but also smaller than the ball; therefore they both have the same amount of clay, etc.). When the child gave either an incorrect judgment or an incorrect justification, a score of 0 was obtained.

\section{Procedure}

The 13 conservation tasks were administered individually by 13 experienced experimenters. In each age level approximately the same number of children was assigned at random to each experimenter. The duration of each test varied according to the motivation and concentration of the child. When the child gave signs that he/she did not want to continue, then the remaining tasks were completed in a following session. Each of the 13 experimenters had more than two years research experience with young children. In addition to this, before beginning the present study, the experimenters received one week of training on administration of the tests.

\section{Results}

\section{Preliminary Analysis}

The homogeneity indices, the KR-20s, of the 13 conservation tasks varied between .94 and .96 for the three groups and the longitudinal group on the three successive test administrations. The item-rest correlations (i.e., item corrected total correlations) ranged from .56 to .88 (with the exception of task $\mathrm{C}$, conservation of distance, with an item-rest correlation of .41 ) on all test administrations. Applying Nunnally's (1978) criteria (KR-20 > .80 and item-rest correlations $\geq .50$ ) it may be concluded that the collection of the 13 conservation tasks may be considered a reliable measure of conservation. 
Table 2

Eigenvalues for the 13 Principal Components of the 13 Conservation Tasks for Each of the Test Administrations

\begin{tabular}{lcccccccccccccc}
\hline & \multicolumn{10}{c}{ Principal Component } \\
\cline { 2 - 13 } & 1 & 2 & 3 & 4 & 5 & 6 & 7 & 8 & 9 & 10 & 11 & 12 & 13 \\
\hline September & 7.28 & .93 & .89 & .79 & .72 & .59 & .52 & .46 & .30 & .20 & .16 & .10 & .04 \\
January & 8.06 & .89 & .82 & .75 & .66 & .57 & .46 & .35 & .23 & .12 & .04 & .03 & .02 \\
April & 8.97 & .82 & .77 & .65 & .59 & .49 & .33 & .23 & .07 & .04 & .03 & .02 & .01 \\
Longitudina1 & & & & & & & & & & & & & \\
$\quad \begin{array}{l}\text { September } \\
\text { January }\end{array}$ & 7.54 & .90 & .88 & .78 & .71 & .62 & .55 & .46 & .32 & .19 & .10 & .04 & .01 \\
$\quad$ April & 8.06 & .87 & .80 & .77 & .68 & .59 & .47 & .34 & .24 & .12 & .08 & .06 & .02 \\
& 8.71 & .85 & .78 & .68 & .59 & .47 & .34 & .22 & .15 & .10 & .07 & .03 & .01 \\
\hline
\end{tabular}

These results were in agreement with findings of previous research in which the same conservation tasks were also used (Kingma, 1983a, 1983b, 1984c; Kingma \& Koops, 1983).

With the use of factor analysis, it can be investigated whether the collection of these 13 conservation tasks is dominated by one factor only in order to decide whether these tasks may be seen as a homogeneous test (see Nunnally, 1978) for conservation. Such an inspection is necessary, because homogeneous items are needed when applying Mokken scale analysis.

Since there are four different age levels in each of the four groups, it might be argued that chronological age (CA) has to be partialled out before applying factor analysis (see, e.g., Humphrey \& Parsons, 1979). In contrast, it can be stated from a developmental point of view, that partialling out CA would not be suitable, because it might "throw out the baby of Piagetian development with the bath of CA" (Kohlberg \& De Vries, 1980, p. 176). Generally, CA has not been partialled out in factor analytic studies on Piagetian batteries (see for a survey, Horn, 1976; Modgil \& Modgil, 1976). In addition, Glass and Stephens (1980) argued that the final factor structure is relatively insensitive to removal of age. In line with these arguments, age was not partialled out in the present study.

Factor analysis (principal components with iteration) of the scores was performed for the score of all children in each of the four groups (whereas for the longitudinal group, it was also investigated for the three successive test administrations). In each of the groups all the first principal components had eigenvalues $\geq 1.0$, whereas the second and following principal components had eigenvalues $<$ 1.0. (Table 2 shows the eigenvalues of the principal components for these conservation tasks on the different administrations.)

The explained variance of the first principal component varied between $56 \%$ and $69 \%$ for the different test administrations. According to the criterion of eigenvalue $\geq 1.0$ (i.e., Kaiser's, 1961, rule), no orthogonal Varimax rotation was carried out (since all 13 tasks had high factor loadings on the first principal component and low factor loadings on the second and following principal components). The same results were obtained by applying Cattell's scree rule (Cattell, 1966; Zwick \& Velicer, 1982). These results were in agreement with previous findings (Kingma, 1983b, 1983c, 1984b, 1984c; Kingma \& Koops, 1983). In sum, it may be concluded that the 13 conservation tasks were both a reliable and a homogeneous test for conservation. Therefore, Mokken scale analysis can be applied to these data.

\section{Scale Analysis}

The coefficients $H$ of scalability (Loevinger, 1948; Mokken, 1971) were computed for the 13 conservation tasks. For the September, January, and April groups $H$ was, respectively, $.65, .73$, and .79, whereas for the longitudinal group $H$ was, respectively, $.68, .73$, and .78 on the three successive test administrations. Thus, the scalability of the items for both types of conservation tasks could be considered very strong $(H>.50$, see Mokken, 
1971). However, great differences were observed for the values of the scalability of the individual items, $H_{i}$, which ranged between .44 and .88 . Also deviations were found in the $\mathbf{P}$ and $\mathbf{P}_{0}$ matrices (Mokken, 1971), in which the values must show, respectively, an increase and a decrease in both the rows and columns. It can be stated that some of the conservation tasks did not meet the criterion of double monotony.

Further inspection of the $\mathbf{P}$ and $\mathbf{P}_{0}$ matrices revealed that for each of the three groups (September, January, and April) both different types and different numbers of conservation tasks violated the assumption of double monotony. The same picture was obtained for the longitudinal group, that is, on the first administration the same conservation tasks did not meet the criterion of double monotony as was observed for the September group; on the second and third administration the same tasks violated the assumption of double monotony as was observed in, respectively, the January and the April group.

Ultimately, the seven tasks selected (which met the assumption of double monotony) were for the September group and for the first administration of the longitudinal group: A (conservation of surface), $B$ (conservation of number), D (conservation of substance), $\mathrm{F}$ (conservation of quantity), $\mathrm{H}$ (conservation of weight), I (conservation of covered surface), and $\mathrm{J}$ (conservation of length). Eight tasks were selected for the January group and for the second administration of the longitudinal group: the seven tasks of the September group and task $K$ (conservation of discontinuous length). The selection of the conservation tasks (which met the requirement of double monotony) consisted of nine tasks both for the April group and the third administration of the longitudinal group: the seven tasks of the September group and tasks G (conservation of discontinuous quantity) and $\mathrm{L}$ (conservation of geometric volume). The coefficients of scalability, $H_{i}$, of the seven selected tasks varied between .74 and .85 for the September group and the first administration of the longitudinal group.

There were no deviations observed for the double monotony for the $\mathbf{P}$ and $\mathbf{P}_{0}$ matrices outside the acceptable range of .03 (see Mokken, 1971). The coefficient $H$ for all seven conservation tasks together was .78 for the September group and .80 for the first administration of the longitudinal group.

In the January group and the second administration for the longitudinal group, the eight selected tasks also formed a strong Mokken scale with $H$ values of .73 and .74 , respectively, which met the assumptions of double monotony. The coefficients of scalability $H_{i}$ of these eight tasks ranged between .72 and .82 .

It can be argued that the scale of both the September group and the first administration of the longitudinal group may be considered a subscale of the scale found for the January group and the second administration of the longitudinal group. However, closer inspection reveals that this is not the case, because tasks $\mathrm{H}$ and $\mathrm{J}$ have reversed positions on the two types of conservation scales (as indicated by the $p$ values of these tasks).

Concerning the April group and the third administration of the longitudinal group, a strong Mokken scale was found for the nine selected tasks, which did not show any deviations of the $\mathbf{P}$ and $\mathbf{P}_{0}$ matrices outside the acceptable range of .03 . The $H$ values were .79 and .78 , respectively, for the April group and the third administration of the longitudinal group. The coefficients of scalability of the tasks, $H_{i}$, varied between .74 and .88 .

Some tasks of the subset of conservation tasksA, B, D, F, H, I, and J-from this scale did not occupy identical positions on those scales found at earlier test administrations. For the April group tasks $\mathrm{A}$ and I showed a reversal to the relative positions of the same tasks on the scales of the September and January group, respectively. This was also observed for tasks D and F. These reversals can also be found for the successive test administrations of the longitudinal group. Thus, the subset of these seven tasks did not provide a stable picture of the acquisition of conservation ability across the three successive test administrations.

In contrast, the scale of the selected tasks for the different groups administered at the same time may be considered more or less identical. However, on the subsequent test administrations the scales contained an increasing number of conservation tasks: seven, eight, and nine tasks, respec- 
tively, for the September group, the January group, and the April group. This might reflect a developmental trend in the acquisition of conservation ability, as suggested by the increasing $p$ values.

Since the scales of the successive administrations of the longitudinal study were similar to the scales found for the samples that had been administered at the same point in time, it was investigated whether the September group and the first administration of the longitudinal group could be combined into one group. This was also analyzed for the January group and the second administration of the longitudinal group. Therefore, an analysis was performed with program Mokken Scale (Niemoller et al., 1980). With the use of this computer program the invariance of scalability can be computed (i.e., the robustness of the scale) for several groups. Therefore, the value of the statistic $T$ has to be computed (see Equation 4).

Under the null hypothesis that the conservation tasks have the same scalability in each of the two groups, $T$ shows an approximate chi-square distribution with 1 degree of freedom. It was found that $T=1.36\left(\chi_{\text {crit }}^{2}=6.64, \alpha=.01\right)$ for the September group and the first administration of the longitudinal group. A similar picture was found for the January group and the second administration of the longitudinal group $(T=.96)$, as well as for the April group and the third administration of the longitudinal group $(T=.06)$. These results demonstrated that the invariance of scalability was very strong for the different groups who were tested at the same point in time. Thus, it can be concluded that the selected conservation tasks had the same scalability for each of the two groups on the three successive test administrations.

Therefore, the September group and the first administration of the longitudinal group were combined into the combined September group $(N=$ 384). The January group and the second administration were placed together in the combined January group $(N=382)$, whereas the April group and the third administration of the longitudinal group were taken together in the combined April group $(N=391)$. For the three combined groups the coefficients of scalability were computed again for the selected tasks in order to base the values of the ultimate scales on three large samples. Table 3 shows the $p$ values and the $H_{i}$ coefficients of the selected tasks for the three combined groups. The coefficients $H$ of scalability of the scales were $.80, .81$, and .79 , respectively, for each of the combined September, January, and April groups.

It can be seen from Table 3, that the $p$ values as well as some of the scalability coefficients of tasks A, B, D, F, H, I, and J showed a slight increase in each of the test administrations. It can be concluded that though the scales were invariant for different samples at the same point in time, these values of scalability can increase during the course of development, as Mokken (1971) has suggested.

The reliability of the tasks of the ultimate three scales were almost equal to values of the 13 original conservation tasks. (KR-20 varied between .91 and .93 for the different test administrations.) Closer inspection reveals that for the selection of the items from the ultimate scales, those items were omitted that had the lowest item-rest correlations. Thus, the analysis of the reliability of the original tasks may provide a rough indication of the kind of tasks that were possible candidates for not fitting on the ultimate scale.

\section{Discussion}

The stochastic Mokken scale analysis was shown to be very useful for constructing a strong scale for conservation tasks. It was shown that the number of selected tasks (which met the assumption of double monotony) increased on the successive test administrations. This finding suggested a developmental trend for conservation as indicated by increasing $p$ values of a subset of the same seven tasks for each of the three types of scales. The slight increase in the number of tasks on the scales from September to April (respectively from seven, eight, to nine), might be interpreted as a gradual extension of conservation ability. However, this extension was not a stable one, because of the January administration task $\mathbf{J}$ (conservation of discontinuous length) fitted on the scale, whereas at the April administration this task could not be selected for the scale since it violated the assumption 
Table 3

Coefficient $\mathrm{H}_{i}$ and the $P$ Values of the Selected Tasks from the Ultimate Scales for the Three Combined Groups

\begin{tabular}{|c|c|c|c|c|c|c|c|}
\hline & \multirow[b]{2}{*}{ Tasks } & \multicolumn{2}{|c|}{$\begin{array}{l}\text { September } \\
(\mathrm{N}=384)\end{array}$} & \multicolumn{2}{|c|}{$\begin{array}{c}\text { January } \\
(\mathrm{N}=382)\end{array}$} & \multicolumn{2}{|c|}{$\begin{array}{c}\text { April } \\
(\mathrm{N}=391)\end{array}$} \\
\hline & & $\mathrm{H}_{\mathbf{i}}$ & $P$ & $\mathrm{H}_{\mathrm{i}}$ & $\mathrm{P}$ & $\mathrm{H}_{\mathbf{i}}$ & $P$ \\
\hline A & $\begin{array}{l}\text { conservation of } \\
\text { surface }\end{array}$ & .82 & .21 & .81 & .25 & .88 & .37 \\
\hline B & conservation of & & & & & & \\
\hline & number & .85 & .52 & .78 & .59 & .89 & .63 \\
\hline D & conservation of & & & & & & \\
\hline$F$ & $\begin{array}{l}\text { substance } \\
\text { conservation of }\end{array}$ & .80 & .40 & .81 & .44 & .85 & .49 \\
\hline & quantity & .79 & .38 & .79 & .43 & .85 & .52 \\
\hline G & $\begin{array}{l}\text { conservation of } \\
\text { discontinuous }\end{array}$ & & & & & & \\
\hline $\mathrm{H}$ & $\begin{array}{l}\text { quantity } \\
\text { conservation of }\end{array}$ & - & - & - & -- & .88 & .54 \\
\hline & weight & .74 & .31 & .73 & .33 & .76 & .44 \\
\hline I & $\begin{array}{l}\text { conservation of } \\
\text { covered surface }\end{array}$ & .81 & .23 & .82 & .28 & .87 & .36 \\
\hline $\mathrm{K}$ & $\begin{array}{l}\text { conservation of } \\
\text { length } \\
\text { conservation of } \\
\text { discontinuous }\end{array}$ & .74 & .31 & .76 & .38 & .83 & .46 \\
\hline L & $\begin{array}{l}\text { length } \\
\text { conservation of }\end{array}$ & -- & - & .78 & .35 & -- & -- \\
\hline & geometric volume & - & -- & -- & -- & .78 & .42 \\
\hline
\end{tabular}

of double monotony. Thus, due to the development of conservation during the periods between the three test administrations, the order of acquisition of different conservation concepts appeared to fluctuate somewhat. This finding suggests that for comparing results of conservation experiments, the point in time of the test administration has to be taken into account.

It was also shown that the scales of these tasks were invariant for different samples tested at the same point in time. Therefore, the September group and the first administration of the longitudinal group could be combined. Also the January group and the second administration of the longitudinal group could be taken together, as well as the April group and the third administration of the longitudinal group. Thus, the ultimate computations of the $p$ values and the coefficients of scalability were based on three groups of subjects, respectively, 384 (com- bined September group), 382 (combined January group), and 391 (combined April group). The reliability of the selected tasks of the three ultimate scales was almost equal to the value found for the 13 original conservation tasks. Inspection of the item-rest correlations revealed that tasks with the lowest item-rest correlations (in the range of .50 to .60) did not meet the assumptions of double monotony. Thus, the results of the reliability study provided a rough indication of which tasks might not fit in the ultimate scale.

Because the selected tasks formed a strong Mokken scale (i.e., $H>.50$, see Mokken, 1971), the tradition of developmental psychologists to arrange items according to increasing difficulty obtained an empirical basis in the present study. The advantages of the found scales for conservation is that with these scales the child's level of conservation development (as measured by that scale) can be 
assessed individually. When a child is able to solve one of the most difficult tasks, A (conservation of surface) or I (conservation of covered surface) on these scales, then he/she shows a higher level of development than a child who only reached a correct solution on task B (conservation of number). According to the Mokken model, it can be stated that the former child has a high probability of solving the easier tasks on three scales correctly, whereas the latter child has a low probability of solving one of the more difficult tasks correctly. It can be concluded that nonparametric Mokken scale analysis might be considered a very useful technique for developmental psychological research.

\section{References}

Brainerd, C. J. (1978). Piaget's theory of intelligence. Englewood Cliffs NJ: Prentice Hall.

Brainerd, C. J. (1985). Model approaches to storage and retrieval development. In C. J. Brainerd \& M. Pressley (Eds.), Basic processes in memory development: Progress in cognitive development research (pp. 143207). New York: Springer-Verlag.

Cattell, R. B. (1966). The scree test for the number of factors. Multivariate Behavioral Research, 1, 245276.

Glass, G. V., \& Stephens, B. (1980). Reply to Humphrey's and Parson's Piagetian task measure intelligence and intelligence tests assess cognitive development. Intelligence, 4, 171-174.

Goldschmid, M. L., \& Bentler, P. M. (1968). Manual for the conservation assessment kit. San Diego: Educational and Industrial Testing Service.

Goodman, L. A. (1959). Simple statistical methods for scalogram analysis. Psychometrika, 24, 29-43.

Guttman, L. (1950). The basis for scalogram analysis. In S. S. Stouffer (Ed.), Measurement and prediction (pp. 312-361). Princeton NJ: Princeton University Press.

Hambleton, R. K., \& van der Linden, W. J. (1982). Advances in item response theory and applications: An introduction. Applied Psychological Measurement, 6, 373-378.

Horn, J. L. (1976). Human abilities: A review of research and theory in the early 1970's. In M. R. Rosenzweig \& L. W. Porter (Eds.), Annual Review of Psychology (Vol. 27, pp. 145-163). Palo Alto CA: Annual Reviews.

Humphrey, L. G., \& Parsons, C. K. (1979). Piagetian tasks measure intelligence and intelligence tests assess cognitive development: A reanalysis. Intelligence, 3, 369-382.

Kaiser, H. F. (1961). A note on Guttman's lower bound for the number of common factors. Journal of Statistical Psychology, 14, 175-177.

Kingma, J. (1983a). The development of seriation, conservation and multiple classification. Genetic Psychology Monographs, 108, 43-67.

Kingma, J. (1983b). Piagetian tasks and traditional intelligence as predictors of performance on addition and subtraction tasks in primary school grades one and two. The Journal of Psychology, 115, 39-53.

Kingma, J. (1983c). Seriation, correspondence and transitivity. Journal of Educational Psychology, 75, 763771.

Kingma, J. (1984a). A comparison of four methods of scaling for the acquisition of early number concept. Journal of General Psychology, 110, 23-45.

Kingma, J. (1984b). Criterion problems in conservation research reconsidered from a psychometric point of view. Journal of General Psychology, 111, 109-129.

Kingma, J. (1984c). Traditional intelligence, Piagetian tasks and initial arithmetic in kindergarten and primary school grade one. Journal of Genetic Psychology, 145, 49-60.

Kingma, J., \& Koops, W. (1983). Piagetian tasks, traditional intelligence and achievement tests. British Journal of Educational Psychology, 53, 278-290.

Kingma, J., \& Roelinga, U. (1984). Task sensitivity and the sequence of development in seriation, ordinal correspondence and cardination. Genetic Psychology Monographs, 110, 181-205.

Kohlberg, L., \& De Vries, R. (1980). Don't throw out the Piagetian baby with the psychometric bath: Reply to Humphrey and Parson. Intelligence, 4, 175-177.

Kofsky, F. (1966). A scalogram study of classificatory development. Child Development, 37, 191-204.

Loevinger, J. (1947). A systematic approach to the construction and evaluation of test ability. Psychological Monographs, 61, No. 4.

Loevinger, J. (1948). The technique of homogeneous tests compared with some aspects of 'scale analysis' and factor analysis. Psychological Bulletin, 45, 507530.

Michie, S. (1985). Development of absolute and relative concepts of number in preschool children. Developmental Psychology, 21, 247-252.

Modgil, S., \& Modgil, C. (1976). Piagetian research: Compilation and commentary (Vol. 4). Windsor, G. B.: NFER Publishing Co.

Mokken, R. J. (1971). A theory and procedure of scale analysis. The Hague: Mouton.

Mokken, R. J., \& Lewis, C. (1982). A non-parametric approach to the analysis of dichotomous item responses. Applied Psychological Measurement, 6, 417444. 
Niemoller, B., Verschuur, W., \& Stokman, F. R. (1980). Stochastic cumulative scaling, STAP user's manual, (Vol. 4). Amsterdam, The Netherlands: The University of Amsterdam.

Nunnally, J. C. (1978). Psychometric theory. New York: McGraw-Hill.

Piaget, J. (1952). Logic and psychology. Manchester: Manchester University Press.

Piaget, J., \& Inhelder, B. (1941). Le développement des quantites physiques chez l'enfant. Neuchâtel, Switzerland: Delachaux et Niestlé.

Piaget, J., Inhelder, B., \& Szeminska, A. (1948). La géometrie spontanée de l'enfant. Paris: Presses Universitaires de France.

Piaget, J., \& Szeminska, A. (1941). La genèse du nombre chez l'enfant. Neuchâtel, Switzerland: Delachaux et Niestlé.

Rushton, J. P., Brainerd, C. J., \& Pressley, M. (1984). Behavioral development and construct validity: The principle of aggregation. Psychological Bulletin, 94, 18-38.

Siegel, L. S. (1971). The sequence of development of certain number concepts in preschool children. Developmental Psychology, 5 (2), 357-361.

Vingh Bang (1965). Intuition geometrique et deduction operatoires. Etudes d'Epistemologie Genetique
(Vol. 19). Paris: Presses Universitaires de France.

Wohlwill, J. T. (1960). A study of the development of the number concept by scalogram analysis. The Journal of Genetic Psychology, 97, 345-377.

Zwick, W. R., \& Velicer, W. F. (1982). Factors influencing four rules for determining the number of components to retain. Multivariate Behavioral Research, $17,253-269$.

\section{Acknowledgments}

This research was partially supported by the Netherlands Organization for the Advancement of Pure Scientific Research (Z.W.O. Grant No. S56-177) and partially by a Killam Memorial Award from the University of Alberta. The authors thank two anonymous reviewers for their helpful comments on an earlier draft of this paper.

\section{Author's Address}

Send requests for reprints or further information to Johannes Kingma, Department of Psychology, Biological Sciences Building, University of Alberta, Edmonton, Alberta, Canada, T6G 2E9. 